\title{
Time-Varying Modulated Lapped Transforms
}

\author{
Jelena Kovačević \\ Signal Processing Research Department \\ AT\&T Bell Laboratories \\ Murray Hill, NJ, 07974
}

\author{
Martin Vetterli \\ EECS Department \\ University of California at Berkeley \\ Berkeley, CA, 94720
}

\begin{abstract}
We consider the problem of time-varying orthonormal tilings of the time-frequency plane. A very elegant way of obtaining such tillings is by using time-varying modulated lapped transforms. We offer ways of constructing these, by using either boundary or overlapping modulated lapped transforms. The advantage of using modulated lapped transforms is that all the filters, both at transitions and within decompositions, are obtained by modulation.
\end{abstract}

\section{Introduction}

Recently, there has been a renewal of interest in linear expansions of signals. In particular, wavelets have been used [1], since they can provide a better timefrequency trade-off than the short-time Fourier transform (see Figures 1(a) and (b)). An elegant generalization, which, at least conceptually, contains both the short-time Fourier transform and the wavelet tilings as special cases, is the idea of wavelet packets [2]. They are characterized by their ability to perform an "arbitrary" frequency split, depending on the signal. However, they do not change over time (see Figure 1(c)).

An even better generalization would be if we could find an arbitrary orthonormal tiling (or a biorthonormal in general), that would also vary with time (see Figure $1(\mathrm{~d})$ ). This would allow us to achieve a better representation and compression of nonstationary signals [3]. The approach in this paper is to consider modulated lapped transforms, introduced by Malvar [4]. They can be conceptually seen as duals of wavelet packets (see Figure 2). Since the wavelets are a special case of wavelet packet tilings, time-varying modulated lapped transforms contain duals of wavelets as well. We construct these dual tilings, by using either boundary or overlapping modulated lapped transforms. By boundary modulated lapped transforms, we denote the time-varying tiling of the time-frequency plane where the basis functions do not overlap, while the opposite is true for overlapping modulated lapped transforms, that is, the basis functions from adjacent decompositions do overlap. The advantage of using the modulated lapped transforms (as opposed to more general tilings) is that all the filters, both at transitions, and within decompositions, are obtained by modulation. Note that similar results have been independently obtained in $[5,6]$.

\section{Modulated Lapped Transforms}

By modulated lapped transforms (see, for example, $[4,7])$, we will denote a class of perfect reconstruction filter banks which uses a single prototype filter, window, $w(n)$ of length $2 N$ (where $N$ is the number of channels and is even) to construct all of the filters $h_{0}, \ldots, h_{N-1}$ as follows:

$$
h_{k}(n)=\frac{1}{\sqrt{N}} w(n) \cdot \cos \left(\frac{2 k+1}{4 N}(2 n-N+1) \pi\right)
$$

with $k=0, \ldots, N-1, n=0, \ldots, 2 N-1$, and where the prototype lowpass filter $w(n)$ is symmetric $(w(n)=w(2 N-1-n), n=N, \ldots, 2 N-1)$ and satisfies the following $[7]$ :

$$
w^{2}(n)+w^{2}(N-1-n)=2, \quad n=0, \ldots, N-1 .
$$

This last condition, imposed on the window, ensures that the resulting modulated lapped transform is orthogonal. The two symmetric halves of the window are called "tails".

In the filter bank literature, there exists a convenient way of analyzing filter banks in time domain, via infinite matrices. Such a matrix $\mathbf{T}$, denotes the transformation from the input signal into the interleaved subband signals (for more details, see, [8]). For modulated lapped transforms, the matrix $T$ can be 

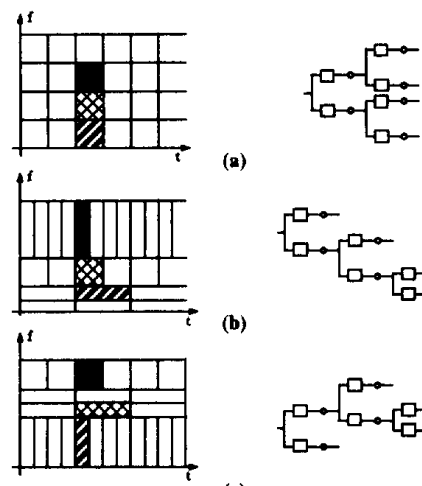

ra-

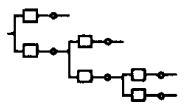

(b)

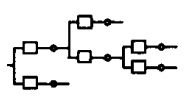

(c)
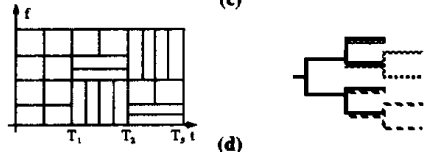

Figure 1: Tilings of the time-frequency plane. (a) Short-time Fourier transform tiling. (b) Wavelet tiling. (c) Wavelet packet tiling. (d) Generalized tiling which adapts in time as well as in frequency. From $\left(0, T_{1}\right)$ the tiling is as in (a) and is denoted by the solid black line one the left. From $\left(T_{1}, T_{2}\right)$, the tiling is as in (c) and is denoted by the grey line, while from $\left(T_{2}, T_{3}\right)$, the tiling is as in (b) and is denoted by the dashed line on the left.

written as

$$
\mathbf{T}=\left[\begin{array}{lllll}
\ddots & & & & \\
& \mathbf{A}_{0} & \mathbf{A}_{1} & & \\
& & \mathbf{A}_{0} & \mathbf{A}_{1} & \\
& & & & \ddots
\end{array}\right],
$$

where blocks $\mathbf{A}_{0}, \mathbf{A}_{1}$ are of sizes $N \times N$, and contain the impulse responses of the filters. Note that the filter length is twice the number of channels. For example, the $j$ th row of $\mathbf{A}_{i}$ is $\left[h_{j}(2 N-1-i N) \ldots h_{j}(N-i N)\right]$ for $i=0,1$. For an orthogonal, perfect reconstruction solution, the matrix $\mathbf{T}$ has to be unitary, which is equivalent to the following [8]:

$$
\mathbf{A}_{0} \mathbf{A}_{0}^{T}+\mathbf{A}_{1} \mathbf{A}_{1}^{T}=\mathbf{I}, \quad \mathbf{A}_{1} \mathbf{A}_{0}^{T}=\mathbf{0}, \quad \mathbf{A}_{0} \mathbf{A}_{1}^{T}=\mathbf{0} .
$$

The second and third conditions in the above are called the "orthogonality of tails" conditions [8].

We want to derive now an interesting fact, that will be used in later constructions. In the matrix $\mathbf{T}$, denote by $w_{A}$ the window function corresponding to the block $\mathbf{A}_{0}$. Take the same block, that is, $\mathbf{A}_{0}$, and
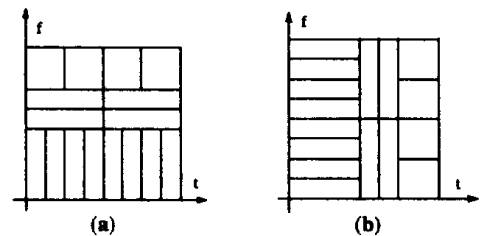

(b)
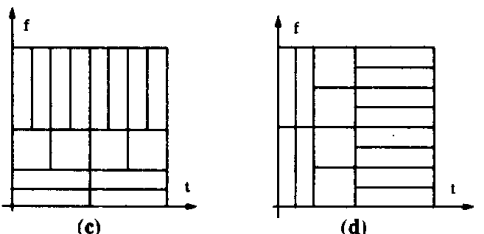

(d)

Figure 2: (a) Wavelet packets have their dual in (b) time-varying modulated lapped transforms. (c) Wavelet tiling has its dual in (d) a particular class of time-varying modulated lapped transforms.

change the window function to $w_{B}$. Call this newly obtained block $\mathbf{B}_{0}$. Then

$$
\mathbf{B}_{0} \mathbf{B}_{0}^{T}=\mathbf{A}_{0} \mathbf{A}_{0}^{T},
$$

that is, the product $\mathbf{A}_{0} \mathbf{A}_{0}^{T}$ does not depend on the window (the proof of which can be found in [3]). The same is true of the other block $\mathbf{A}_{1}$. An interesting consequence of the above, is that

$$
\begin{aligned}
& \mathbf{B}_{0} \mathbf{B}_{0}^{T}+\mathbf{A}_{1} \mathbf{A}_{1}^{T}=\mathbf{I} \\
& \mathbf{A}_{0} \mathbf{A}_{0}^{T}+\mathbf{B}_{1} \mathbf{B}_{1}^{T}=\mathbf{I} \\
& \mathbf{A}_{0} \mathbf{A}_{0}^{T}+\mathbf{A}_{1} \mathbf{A}_{1}^{T}=\mathbf{I}
\end{aligned}
$$

or, in other words, any combination of blocks with different window "tails" will be unitary, or, we will have a nonsymmetric window. Note, however, that this is not sufficient for a valid orthogonal transform, since the "orthogonality of tails" conditions from (4) does not hold, namely $\mathbf{A}_{0} \mathbf{B}_{1}^{T} \neq \mathbf{0}$ (similarly for $\mathbf{B}_{0} \mathbf{A}_{1}^{T}$ ). Nevertheless, the fact that $\left[\mathbf{A}_{0} \mathbf{B}_{1}\right]$ is unitary despite $\mathbf{A}_{0}$ and $\mathbf{B}_{1}$ having different windows, is going to be very useful in constructing boundary and overlapping modulated lapped transforms.

\section{Boundary Modulated Lapped Trans- forms}

Let us now try to construct a set of boundary filters for the modulated lapped transforms. From the results in $[3,9]$, we know that we can always do this by 
applying the Gram-Schmidt procedure to the appropriately truncated matrix $\mathbf{T}$. However, our approach here is different; namely, we want the boundary filters to be obtained by modulation as well. Therefore, consider the following matrix:

$$
\mathbf{T}_{b}=\left[\begin{array}{ccccc}
\mathbf{B}_{0} & \mathbf{A}_{1} & & & \\
& \mathbf{A}_{0} & \mathbf{A}_{1} & & \\
& & \ddots & & \\
& & \mathbf{A}_{0} & \mathbf{A}_{1} & \\
& & & \mathbf{A}_{0} & \mathbf{B}_{1}
\end{array}\right],
$$

where $\mathbf{A}_{i}$ are size- $(N \times N)$ blocks as introduced in (3), and $\mathbf{B}_{i}$ are size- $(N \times N / 2)$ blocks with the associated window

$$
w_{B}(n)=\left\{\begin{array}{cl}
\sqrt{2} & n=N / 2, \ldots, 3 N / 2-1, \\
0 & \text { otherwise }
\end{array}\right.
$$

For example, the $i$ th row of $\mathbf{B}_{j}$ is given by $\left[h_{j_{B}}((3 / 2) N-1) \ldots h_{j_{B}}(N)\right]$, where $h_{j_{B}}(n)$ is as given in (1) with the window $w_{B}(n)$. If we would use the method in $[3,9]$ to construct boundary filters, we would use $\mathbf{A}_{0}$ in $\mathbf{T}_{b}$ as the left boundary, and then truncate it. Here, on the other hand, we have performed the truncation by using a shorter window (of length $N$ instead of $2 N$ ). The net result is a square matrix, for which we only have to check whether it is unitary. Using (4) and (6)-(8), it can be easily checked that $\mathbf{T}_{b} \mathbf{T}_{b}^{T}=\mathbf{I}$, holds.

Therefore, we have demonstrated how to construct boundary filters for a given modulated lapped transform, so as to be able to change decompositions over time. The transform is still orthogonal, as demonstrated by the fact that $\mathbf{T}_{b} \mathbf{T}_{b}^{T}=\mathbf{I}$. There are still $N$ boundary filters, of length $3 N / 2$. The way they are obtained is by using a nonsymmetric window (which was shown to be possible in the last section).

\section{Overlapping Modulated Lapped Transforms}

Suppose now that we want to be able to switch between two different modulated lapped transforms, but with filters (basis functions) that overlap. We will first show how to change between two same-size modulated lapped transforms but with different windows, and then we will examine the case of switching between different-size transforms.

\subsection{Same-Size Transforms}

Let us now demonstrate how one can switch between two same-size modulated lapped transforms with different windows. Consider the following matrix:

$$
\mathbf{T}=\left[\begin{array}{llllllll}
\ddots & & & & & & & \\
& \mathbf{B}_{0} & \mathbf{B}_{1} & & & & & \\
& & \mathbf{B}_{0} & \mathbf{B}_{1} & & & & \\
& & & \mathbf{B}_{0} & \mathbf{A}_{1} & & & \\
& & & \mathbf{A}_{0} & \mathbf{A}_{1} & & \\
& & & & \mathbf{A}_{0} & \mathbf{A}_{1} & \\
& & & & & & & \ddots
\end{array}\right]
$$

Here, blocks $\left[\begin{array}{lll}\mathbf{B}_{0} & \mathbf{B}_{1}\end{array}\right]$ represent the $N$-channel modulated lapped transform with the window $w_{B}$, while $\left[\begin{array}{ll}\mathbf{A}_{0} & \mathbf{A}_{1}\end{array}\right]$ represent the $N$-channel transform with the window $w_{A}$. It is easily checked that the above matrix is unitary, leading to an orthogonal transform (even across the transition). Note how the transition $\left[\begin{array}{ll}\mathbf{B}_{0} & \mathbf{A}_{1}\end{array}\right]$ uses a nonsymmetric window (or half of the window $w_{B}$ and half of the window $w_{A}$ ) to achieve a transition that is still orthogonal.

\subsection{Different-Size Transforms}

We will now explain how to switch between different-size transforms. Note that a similar result, without details of the construction or the proof, was mentioned in [6].

Here, we want to show that one can switch from an $N_{1}$-channel modulated lapped transform to an $N_{2^{-}}$ channel modulated lapped transform (where $N_{1}<N_{2}$ and $N_{1}=2^{n_{1}}, N_{2}=2^{n_{2}}$ ) as follows:

1. Since the maximum overlap between the two modulated lapped transforms is $N_{1}$, one has to adjust the size of the window of the $\mathrm{N}_{2}$-channel modulated lapped transform accordingly, that is, its length has to be reduced to $N_{2}+N_{1}$, or the outer $\left(N_{2}-N_{1}\right) / 2$ coefficients of each window tail have to be zero. This means that the $\mathrm{N}_{2}$-channel transform can be expressed as follows:

$$
\begin{gathered}
{\left[\begin{array}{lll}
\mathbf{A}_{0} & \mathbf{A}_{1}
\end{array}\right]=} \\
{\left[\begin{array}{llllll}
\mathbf{0} & \mathbf{A}_{00} & \mathbf{A}_{01} & \mathbf{A}_{11} & \mathbf{A}_{10} & \mathbf{0}
\end{array}\right],}
\end{gathered}
$$

where the blocks $\mathbf{A}_{0}$ and $\mathbf{A}_{1}$ have been split into 3 subblocks each, to account for the fact that some of the coefficients of the window are set to 0 . Therefore, the blocks $\mathbf{A}_{01}$ and $\mathbf{A}_{11}$ are those where the window is constant. The blocks $\mathbf{0}$ and $\mathbf{A}_{01}, \mathbf{A}_{11}$ are of size $\left(N_{2}-N_{1}\right) / 2$, while $\mathbf{A}_{00}, \mathbf{A}_{10}$ are of size $N_{1}$.

2. Then, construct the $N_{1}$-channel modulated lapped transform $\left[\begin{array}{ll}\mathbf{A}_{00}^{\prime} & \mathbf{A}_{10}^{\prime}\end{array}\right]$ from the $N_{2}$-channel one 
as follows:

$$
\mathbf{T}_{o}=\left[\begin{array}{cccccc}
\ddots & & & & & \\
\mathbf{A}_{00}^{\prime} & \mathbf{A}_{10}^{\prime} & & & & \\
& \mathbf{A}_{00}^{\prime} & \mathbf{A}_{10}^{\prime} & & & \\
& & \mathbf{A}_{00} & \mathbf{A}_{01} & \mathbf{A}_{11} & \mathbf{A}_{10} \\
& & & & & \mathbf{A}_{00} \\
& & & & & \ddots
\end{array}\right]
$$

where the blocks $\mathbf{A}_{00}^{\prime}$ and $\mathbf{A}_{10}^{\prime}$ are of size $N_{1} \times N_{1}$ and are obtained from the blocks $\mathbf{A}_{00}$ and $\mathbf{A}_{10}$ by scaling by $\sqrt{N_{2} / N_{1}}$ and retaining $N_{1}$ rows with the indices in the following set:

$S=\left\{i \cdot \frac{2 N_{2}}{N_{1}},(i+1) \cdot \frac{2 N_{2}}{N_{1}}-1\right\}, \quad i=0, \ldots, \frac{N_{1}}{2}-1$.

Therefore, if we denote by $h_{k}, g_{k}$ the basis functions of the the $N_{2} / N_{1}$-channel modulated lapped transforms, respectively

$$
g_{k}(n)=\sqrt{\frac{N_{2}}{N_{1}}} h_{k^{\prime}}\left(n+\frac{N_{2}-N_{1}}{2}\right),
$$

for $n \in\left[0, N_{1}-1\right]$ and

$$
g_{k}(n)=\sqrt{\frac{N_{2}}{N_{1}}} h_{k^{\prime}}\left(n+\frac{3 N_{2}-N_{1}}{2}\right)
$$

for $n \in\left[N_{1}, 2 N_{1}-1\right]$ and $\boldsymbol{k}^{\prime} \in S$.

The proof that the above construction indeed leads to an orthogonal transform with overlapping basis functions, is given in the Appendix.

As a simple example, refer to Figure 3(a), where we want to switch between a 2-channel and a 4-channel modulated lapped transform. Thus, we first shorten the window of the 4-channel modulated lapped transform to length 6 as follows:

$$
w(n)=\left\{\begin{array}{cc}
0 & n \in\{0,7\}, \\
\sqrt{2} & n \in\{3,4\}, \\
a & n \in\{1,6\}, \\
\sqrt{2-a^{2}} & n \in\{2,5\}
\end{array}\right.
$$

and choose $S=\{0,3\}$. Then one obtains

$$
g_{k}(n)= \begin{cases}\sqrt{2} h_{k^{\prime}}(n+1) & n \in\{0,1\} \\ \sqrt{2} h_{k^{\prime}}(n+5) & n \in\{2,3\}\end{cases}
$$

with $k=0,1$ and $k^{\prime}=0,3$. Figures $3(\mathrm{~b})$-(e) show another simple example, that is, a switch from a 2channel to an 8-channel modulated lapped transform. A few possibilities are explored: (1) switch directly ending up with 1 degree of freedom, (2) use a nonsymmetric 4-channel transform to obtain a transition, (3) the same as in (2) except that the transition is followed by 4-channel transforms for a while, and (4) avoid a nonsymmetric transform and use 4-channel transforms instead.

An example on a synthetic 512-point test signal can be found in [3], where time-varying modulated lapped transforms of sizes 8,16 and 32 were used. There, one can see that more "stationary" parts of the signal get better frequency resolution, while more uncorrelated parts get better time resolution.
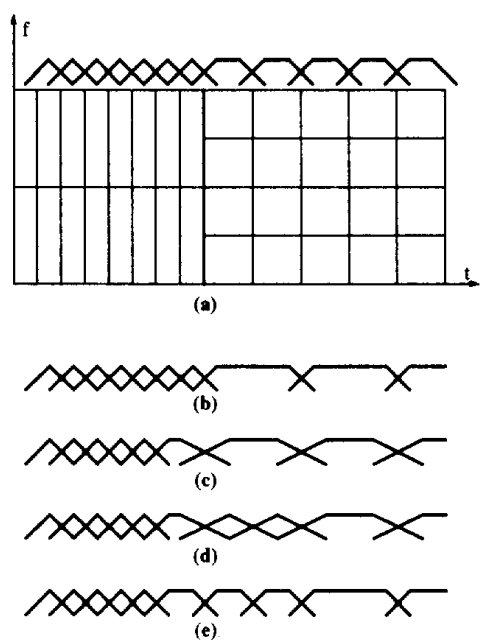

Figure 3: (a) Switching from a 2-channel to an 4channel modulated lapped transform. The thick line denotes the switching point. On top of the tiling, basis functions with appropriate overlaps are given. (b) Example of switching from a 2-channel to an 8-channel modulated lapped transform. Windows with appropriate overlaps are given. Direct switching according to the theorem resulting in only one degree of freedom for designing the window. (c) Use of a transition (4-channel modulated lapped transform) allowing for longer window in the 8-channel modulated lapped transform. (d) Use of transition and 4-channel modulated lapped transforms. (e) Avoiding the transitions by using 4-channel modulated lapped transforms. All the windows are symmetric and have the same tails.

\section{Conclusion}

We have shown how to construct bases which can be seen as duals both of wavelets and wavelet packets. This was achieved by using time-varying modulated lapped transforms, with both overlapping and 
nonoverlapping basis functions. The constructions we gave are, however, more restricted than in the wavelet packets case. In particular, the filter length (basis functions) is restricted to twice the number of channels. On the other hand, the advantage of these timevarying modulated lapped transforms is the existence of a fast algorithm together with the fact that all the filters involved are obtained by modulation.

\section{A Construction of Overlapping Modu- lated Lapped Transforms}

To check that the construction is valid, one has first to prove that the orthogonality of tails for the $N_{1}$ channel bank holds. To prove that, remember that the $\mathrm{N}_{2}$-channel bank is perfect reconstruction by assumption, and thus, orthogonality of its tails holds, together with the fact that all of its basis functions are unitary. The tails in the $N_{1}$-channel case, are obtained from the tails of the $\mathrm{N}_{2}$-channel bank, and thus, by construction, they will be orthogonal to each other (since the tails in the $N_{2}$-channel bank are). Also by construction, the overlapping tails of the $N_{1}$ - and $N_{2}$ channel banks will be orthogonal. The two facts left to show are that the resulting vectors from the $N_{1}$ channel modulated lapped transform are unitary, as well as that they are mutually orthogonal. Call the vectors from the $N_{1}$-channel modulated lapped transform, $\mathrm{g}_{k}$, according to (10) and (11). Then

$$
\mathbf{g}_{k}^{T} \cdot \mathbf{g}_{k}=\frac{N_{2}}{N_{1}}\left(\mathbf{h}_{k^{\prime}}^{T} \cdot \mathbf{h}_{k^{\prime}}-\sum_{n=\left(N_{1}+N_{2}\right) / 2}^{\left(3 N_{2}-N_{1}\right) / 2} h_{k^{\prime}}^{2}(n)\right),
$$

where $\mathbf{h}_{k^{\prime}}$ is the vector from the $N_{2}$-channel bank corresponding to the one from the $N_{1}$-channel one, and, since $N_{2}$-channel bank is perfect reconstruction $\mathbf{h}_{k}^{T} \cdot \mathbf{h}_{k^{\prime}}=1$. The sum term in parentheses is

$$
\begin{gathered}
\sum_{n=\left(N_{1}+N_{2}\right) / 2}^{\left(3 N_{2}-N_{1}\right) / 2} h_{k^{\prime}}^{2}(n)= \\
=\frac{2}{N_{2}} \sum_{n=\left(N_{1}+N_{2}\right) / 2}^{\left(3 N_{2}-N_{1}\right) / 2} \cos ^{2}(\underbrace{\frac{2 k+1}{4 N_{2}} \pi\left(2 n-N_{2}+1\right)}_{x}), \\
=\frac{2}{N_{2}} \sum_{n=\left(N_{1}+N_{2}\right) / 2}^{N_{2}-1}\left(\cos ^{2}(x)+\cos ^{2}\left(\frac{2 k+1}{2} \pi-x\right)\right), \\
=\frac{2}{N_{2}} \sum_{n=\left(N_{1}+N_{2}\right) / 2}^{N_{2}-1}\left(\cos ^{2}(x)+\sin ^{2}(x)\right), \\
=\frac{N_{2}-N_{1}}{N_{2} .}
\end{gathered}
$$

Substituting this into (12), we obtain

$$
\mathbf{g}_{k}^{T} \cdot \mathbf{g}_{k}=\frac{N_{2}}{N_{1}}\left(1-\frac{N_{2}-N_{1}}{N_{2}}\right)=1,
$$

and thus, the vectors are unitary. To prove that they are mutually orthogonal, one has to form all the products $\mathbf{g}_{i}^{T} \cdot \mathbf{g}_{j}, i \neq j$. After some manipulations, and with indices in the range as given in (9), it can be shown that all of these products are zero. $\square$

\section{References}

[1] I. Daubechies, Ten Lectures on Wavelets. Philadelphia, PA: SIAM, 1992.

[2] R. Coifman, Y. Meyer, and V. Wickerhauser, "Wavelet analysis and signal processing," in Wavelets and their Applications (M. B. Ruskai et al., ed.), (Boston, MA), pp. 153-178, Jones and Bartlett, 1992.

[3] C. Herley, J. Kovačević, K. Ramchandran, and M. Vetterli, "Tilings of the time-frequency plane: Construction of arbitrary orthogonal bases and fast tiling algorithms," IEEE Trans. Signal Proc., Special Issue on Wavelets and Signal Processing, vol. 41, December 1993. To appear.

[4] H. Malvar, Signal Processing with Lapped Transforms. Norwood, MA: Artech House, 1992.

[5] R. L. de Queiroz and K. R. Rao, "Time-varying lapped transforms and wavelet packets," IEEE Trans. Signal Proc., Special Issue on Wavelets and Signal Processing, vol. 41, December 1993. To appear.

[6] M. Wickerhauser, "INRIA lectures on wavelet packet algorithms," tech. rep., Dept. of Mathematics, Yale University, March 1991.

[7] J. Princen, A. Johnson, and A. Bradley, "Subband transform coding using filter bank designs based on time domain aliasing cancellation," in Proc. IEEE Int. Conf. Acoust., Speech, and Signal Proc., (Dallas, TX), pp. 2161-2164, April 1987.

[8] M. Vetterli and D. LeGall, "Perfect reconstruction FIR filter banks: Some properties and factorizations," IEEE Trans. Acoust., Speech, and Signal Proc., vol. 37, pp. 1057-1071, July 1989.

[9] C. Herley and M. Vetterli, "Orthogonal timevarying filter banks and wavelet packets," IEEE Trans. Signal Proc., 1993. Submitted. 